OPEN ACCESS

Edited by:

Xiaotian Wang,

Southwest University, China

Reviewed by:

Saadi Berri,

University Ferhat Abbas of

Setif, Algeria

Jabbar M. Khalaf Al-Zyadi,

University of Basrah, Iraq

Fethallah Dahmane,

Centre Universitaire El-Wanchariss

Tissemsilt, Algeria

*Correspondence:

Chuankun Zhang

chknzhang@163.com

Specialty section:

This article was submitted to

Computational Physics,

a section of the journal

Frontiers in Physics

Received: 26 April 2020

Accepted: 28 May 2020

Published: 30 June 2020

Citation:

Zhang C, Huang H, Wu C, Zhu Z, He $Z$ and Liu G (2020) GGA and GGA

Plus $U$ Study of Half-Metallic Quaternary Heusler Compound CoCrScSn. Front. Phys. 8:232. doi: 10.3389/fphy.2020.00232

\section{GGA and GGA Plus U Study of Half-Metallic Quaternary Heusler Compound CoCrScSn}

\author{
Chuankun Zhang*, Haiming Huang, Chengrui Wu, Zhanwu Zhu, Zedong He and \\ Guoying Liu
}

School of Science, Hubei University of Automotive Technology, Shiyan, China

The structural, mechanical, electronic, magnetic, and half-metallic properties of quaternary Heusler compound CoCrScSn are studied using the GGA and GGA + $U$ method based on first-principles calculations. It is found that Type-I structure of CoCrScSn compound is the most stable, and its ground state is ferromagnetic. At the equilibrium lattice constant, the electronic structures obtained by GGA and GGA + $U$ methods indicate that CoCrScSn compound have typical half-metal character. The results of elastic constants and half-metallic robustness show that the mechanical stability and half-metallicity of CoCrScSn can be well-maintained in the range of 6.2-6.9 $\AA$ under GGA and 5.7-6.4 ̊ under GGA + U, respectively. When CoCrScSn compound exhibits half-metallic properties, the total magnetic moment per molecular unit is 4.0 $\mu_{\mathrm{B}}$, which is in good agreement with the Slater-Pauling rule, and $\mathrm{Cr}$ atoms are the main source of molecular magnetic moment. All the aforementioned results indicate that quaternary Heusler compound CoCrScSn would be an ideal candidate in spintronics.

Keywords: heusler, half-metal, elastic constant, first-principles, spintronics

\section{INTRODUCTION}

Spintronics, also known as magnetoelectronics, is a new discipline and technology that has developed rapidly since the 1990s [1,2]. The information transport of traditional electronic devices mainly depends on the charge. Spintronics not only uses the charge transport, but also adds the spin and magnetic moment of the electron. Compared with traditional semiconductor electronic devices, spintronic devices have many advantages such as non-volatile, low power consumption and high integration. For spintronic materials, it is necessary to have higher electron polarizability and in this case, half-metallic magnetic materials become the best choice for spintronic applications. In the field of spintronics, half-metallic magnetic materials are widely used in spin diodes, spin valves, spin filters, and other devices due to their special electronic structure [3-5]. The most important feature of half-metallic magnetic materials is that the majority-spin (minority-spin) direction is metal property, and the minority-spin (majority-spin) direction is semiconductor or insulator property, leading to $100 \%$ spin polarization at the Fermi level $[6,7]$. The unique electronic structure of half-metallic magnetic materials has become a research hotspot recently [8-10].

Heusler alloy compounds have always been one of the most important members of half-metallic magnetic materials. Since the first half-metallic magnetic material, ternary Heulser alloy NiMnSb [11] was predicted, researchers have paid great attention to the this kinds of compounds [12-25]. The crystal of Heusler compounds is generally cubic structure with four atoms. The four atoms occupy $\mathrm{A}(0,0,0), \mathrm{B}(0.25,0.25,0.25), \mathrm{C}(0.50,0.50,0.50)$, and $\mathrm{D}(0.75,0.75,0.75)$, respectively $[26,27]$. 
According to the different ways of occupying atoms, Heusler compounds have derived many types of structures, including fullHeusler, half-Heuler, inverse-Heusler, and quaternary-Heusler, etc. There are many half-metallic magnetic materials in these different Heusler configurations, and they are widely concerned by researchers in experiments and theory [28-34].

In recent years, there are some reports about the CoCr-based quaternary Heusler compounds. In 2012, Gokoglu [35] found that quaternary Heusler compound $\mathrm{NiCoCrGa}$ displays a slightly disturbed half-metallic behavior with high-spin polarization ratio at Fermi level. In 2017, Xu et al. [36] found that the quaternary Heusler ( $\mathrm{Y}, \mathrm{La}, \mathrm{Lu}) \mathrm{CoCr} / \mathrm{FeMn}(\mathrm{Al}, \mathrm{Ga})$ containing rare earth elements has the characteristics of spin gapless semiconductors. In 2019, Wu et al. [37] found that the Curie temperature of $\mathrm{CrCoScZ}(\mathrm{Z}=\mathrm{Si}, \mathrm{Ge}, \mathrm{Sn})$ based on mean field theory is higher than that of room temperature. Huang et al. [38] found that the bulk and surface states of $\mathrm{CoCrScBi}$ are half-metallic. In 2020, Khan et al. [39] studied the structural stability, band structure, density of states, magnetic and mechanical properties of CoCrbased quaternary Heusler compounds $\operatorname{CoCrYZ~(Z=Si,~Ge,~Ga,~}$ $\mathrm{Al}$ ) and found all these materials possess half-metallic character.

Recent studies on quaternary Heusler compounds show that the Coulomb interaction of $3 \mathrm{~d}$ electrons in transition metals has a significant effect on the electronic structure of alloys. Gao et al. [40] reveal that the half-metallicity is lost for both $\mathrm{CoFeCrAl}$ and $\mathrm{CoFeCrGa}$ but retentive for both $\mathrm{CoFeCrSi}$ and $\mathrm{CoFeCrGe}$ when the Coulomb interactions are considered. Paudel et al. [41] found the band gap width of CoFeZrGe, CoFeZrSb, and CoFeZrSi compounds under GGA $+\mathrm{U}$ changed a lot compared with that under GGA. Although CoCrScSn compound has been shown to have half-metallic behavior in previous studies [37], there has been no report on whether it has stable mechanical properties, nor on whether the Coulomb interactions between transition metal atoms in $\mathrm{CoCrScSn}$ affect its physical properties. Therefore, in order to reveal the effect of the coulomb interaction on the electronic structure of $\mathrm{CoCrScSn}$, and also to understand its mechanical stability, so that $\mathrm{CoCrScSn}$ can be applied in spintronics. In this study, we perform a complete first-principles study of the ground state, mechanical, electronic, magnetic, and half-metallic properties of $\mathrm{CoCrScSn}$ compound based on the generalized gradient approximation (GGA) [42] with strong-correlated correction $(\mathrm{GGA}+\mathrm{U})$.

\section{CALCULATION METHODS}

In this study, the GGA and GGA + $U$ as implemented in the Vienna ab-initio simulation package (VASP) package [43], was used to calculate the physical and mechanical properties of the CoCrScSn compound. The effective $\mathrm{U}\left(\mathrm{U}_{\text {eff }}\right)$ values of $2.7 \mathrm{eV}$ for $\mathrm{Co}, 2.0 \mathrm{eV}$ for $\mathrm{Cr}$, and $0.5 \mathrm{eV}$ for $\mathrm{Sc}[44,45]$ were selected, respectively, as these values provides correct description magnetic moment and electronic structure in the Heusler compounds [46]. For the self-consistent calculation, the plane wave cutoff energy was chosen to be $500 \mathrm{eV}$. A mesh of $9 \times 9 \times$ 9 Monkhorst-Pack k-point was used. The convergence tolerances
TABLE 1 | Equilibrium lattice equilibrium constants $(\AA)$ and atomic positions for three different types of crystals.

\begin{tabular}{lccccc}
\hline Types & a & Co & Cr & Sc & Sn \\
\hline Type-I & 6.3848 & $3 / 4,3 / 4,3 / 4$ & $1 / 4,1 / 4,1 / 4$ & $1 / 2,1 / 2,1 / 2$ & $0,0,0$ \\
Type-II & 6.4172 & $3 / 4,3 / 4,3 / 4$ & $1 / 2,1 / 2,1 / 2$ & $1 / 4,1 / 4,1 / 4$ & $0,0,0$ \\
Type-III & 6.4705 & $3 / 4,3 / 4,3 / 4$ & $0,0,0$ & $1 / 2,1 / 2,1 / 2$ & $1 / 4,1 / 4,1 / 4$ \\
\hline
\end{tabular}

TABLE 2 | Total energies (eV) of CoCrScSn compound in NM, FM, and AFM states.

\begin{tabular}{lccc}
\hline Types & NM & FM & AFM \\
\hline Type-I & -105.1269 & -108.6031 & -108.5538 \\
Type-II & -101.0947 & -105.3795 & -105.0217 \\
Type-III & -102.5495 & -106.5999 & -105.5016
\end{tabular}

were selected as the difference in total energy and the maximum force within $1.0 \times 10^{-5} \mathrm{eV}$ and $1.0 \times 10^{-2} \mathrm{eV} /$ atom, respectively.

\section{RESULTS AND DISCUSSIONS}

\section{Structural Properties}

According to the results of earlier research, quaternary Heusler compounds usually have three different structures depending on the positions occupied by the atoms. The three structures are defined as type-I, type-II, and type-III, respectively [47-50]. For $\mathrm{CoCrScSn}$ compound, the occupancy positions of $\mathrm{Co}, \mathrm{Cr}, \mathrm{Sc}$, and Sn atoms are shown in Table 1. We must first determine which of the three structures is the most stable. Therefore, we are very concerned about the relationship between the total energy of the three structures and the lattice constant. Through structural optimization, we have obtained the equilibrium lattice constants of three structures of CoCrScSn compounds as shown in Table $\mathbf{1 .}$ Table 2 shows the total energies of $\mathrm{CoCrScSn}$ compound in non-magnetic (NM), ferromagnetic (FM), and antiferromagnetic (AFM) states at the equilibrium structures. It can be seen that among these three structures, the energy of the ferromagnetic state with the type-I structure is the lowest, which means that the type-I structure of the $\mathrm{CoCrScSn}$ compound is the most stable, and the ground state of this stable structure is ferromagnetic. The next studies only consider the ferromagnetic type-I structure of CoCrScSn compound.

So far, there are no experimental reports about CoCrScSn compound. In order to test the possibility of synthesizing $\mathrm{CoCrScSn}$ compound, we performed a calculation of formation energy, which is calculated as follows:

$$
E_{f}=E_{T o t}-E_{C o}-E_{C r}-E_{S c}-E_{S n}
$$

where $E_{T o t}$ are the total energy of the CoCrScSn compound per formula unit at equilibrium lattice constants and $E_{C o}, E_{C r}, E_{S c}$, and $E_{S n}$ correspond to the total energy per atom for the $\mathrm{Co}, \mathrm{Cr}$, $\mathrm{Sc}$, and $\mathrm{Sn}$ atoms, respectively. During calculation, $\mathrm{Co}, \mathrm{Cr}$, and Sc are taken as face-centered cubic structure (space group Fm-3m), 


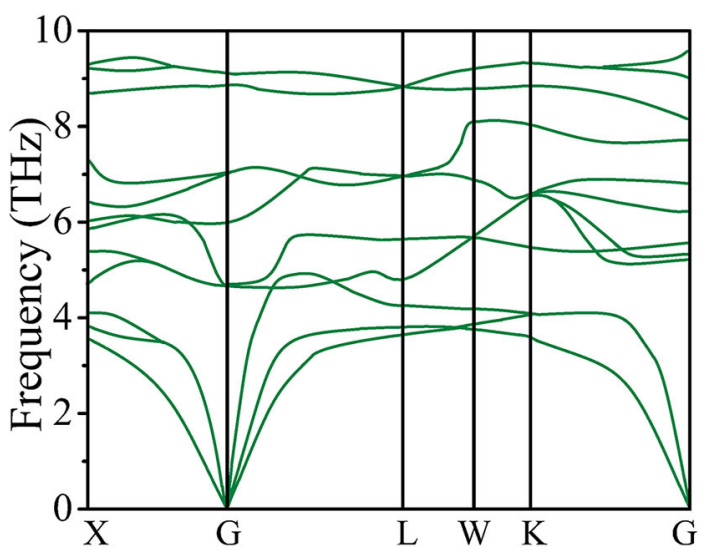

FIGURE 1 | Phonon dispersion curves of the CoCrScSn compound at equilibrium lattice constants.

Sn is taken as body-centered cubic structure (space group Im$3 \mathrm{~m})$. We use the ferromagnetic energy of the title compound as the $E_{T o t}$ of formula (1) to obtain the formation energy of type-I, type-II, and type-III as $-1.35,-0.55$, and $-0.85 \mathrm{eV}$, respectively. The negative values of the formation energy indicate that these three structures of CoCrScSn compounds are energetically stable and may be fabricated experimentally.

In order to examine the dynamical stability, the phonon dispersion curves of the type-I structure of $\mathrm{CoCrScSn}$ compound have been calculated and depicted in Figure 1. It is seen that the title compound have positive phonon frequency, which is an key indicator of the dynamical stability of the Heusle compound. There are no available experimental related to the quaternary Heusler compoud $\mathrm{CoCrScSn}$ to make a comparison with our data, and we are looking forward to the experimental synthesis of the title compound.

\section{Mechanical Properties}

The mechanical properties of cubic crystals are determined by three elastic constants, $\mathrm{C}_{11}, \mathrm{C}_{12}$, and $\mathrm{C}_{44}$. The elastic constants $\mathrm{C}_{11}, \mathrm{C}_{12}$, and $\mathrm{C}_{44}$ at are obtained by calculating the strain as a function of total energy. Born and Huang criteria give that the mechanical stability of cubic crystals is determined by the following formula [51, 52]:

$$
C_{11}-C_{12}>0 C_{44}>0 C_{11}+2 C_{12}>0
$$

The elastic constants $\mathrm{C}_{11}, \mathrm{C}_{12}$, and $\mathrm{C}_{44}$ of CoCrScSn compound at equilibrium lattice constant were calculated to be $154.9 \mathrm{GPa}$, 61.5 GPa, and 62.7 GPa for GGA and 165.2 GPa, 70.3 GPa, and 74.2 GPa for GGA + U, respectively. As a comparison, we found that the $\mathrm{C}_{11}$ and $\mathrm{C}_{44}$ of $\mathrm{CoCrScSn}$ in this paper were larger than that of another similar compound $\mathrm{CoCrScBi}$, while $\mathrm{C}_{12}$ was smaller than that of $\mathrm{CoCrScBi}[38]$.

$$
\begin{array}{r}
B=\frac{C_{11}+2 C_{12}}{3} \\
G_{V}=\frac{C_{11}-C_{12}+3 C_{44}}{5} \\
G_{R}=\frac{5\left(C_{11}-C_{12}\right) C_{44}}{4 C_{44}+3\left(C_{11}-C_{12}\right)} \\
G=\frac{G_{V}+G_{R}}{2}
\end{array}
$$

According to formula (3)-(6), the bulk modulus $B$ and shear modulus $G$ calculated are $92.63 \mathrm{GPa}, 55.72 \mathrm{GPa}$ for GGA, and 101.93 GPa, 62.02 GPa for GGA + U, respectively. The bulk modulus and shear modulus of $\mathrm{CoCrScBi}$ are reported as 127.03 $\mathrm{GPa}$ and $31.88 \mathrm{GPa}$, respectively. The bulk modulus of CoCrScSn is smaller than that of $\mathrm{CoCrScBi}$, indicating that $\mathrm{CoCrScSn}$ is more prone to volume deformation than $\mathrm{CoCrScBi}$. However, the larger shear modulus of $\mathrm{CoCrScSn}$ confirms its greater resistance to shear strain than $\mathrm{CoCrScBi}$. The elastic constants obtained by using GGA and GGA + $U$ all meet the elastic stability criteria and therefore, ferromagnetic type-I structure of $\mathrm{CoCrScSn}$ compound are mechanically stable.

As we all know, the physical properties of compounds will change with the change of crystal structure. The change of lattice constant of compounds under external pressure is one of the simplest ways to change the physical properties of compounds. Next, we study the mechanical stability of $\mathrm{CoCrScSn}$ compound when the lattice constant changes.

Under the uniform pressure $P$, the stress-strain coefficients will replace the elastic constants with the following expression:

$$
B_{11}=C_{11}-P, B_{12}=C_{12}+P, B_{44}=C_{44}-P
$$

Accordingly, the mechanical stability of $\mathrm{CoCrScSn}$ compound under uniform pressure is determined by the following formula $[53,54]$ :

$$
B_{11}-B_{12}>0, B_{11}+2 B_{12}>0, B_{44}>0
$$

That is

$$
C_{11}-C_{12}-2 P>0, C_{11}+2 C_{12}+P>0, C_{44}-P>0
$$

Figures 2A,B shows the change curve of the elastic modulus of $\mathrm{CoCrScSn}$ compound with the lattice constant under GGA and GGA + U, respectively. Under GGA, when the lattice constant of CoCrScSn compound is between 5.8 and $7.2 \AA, \mathrm{C}_{11}-\mathrm{C}_{12}$ $2 \mathrm{P}, \mathrm{C}_{11}+2 \mathrm{C}_{12}+\mathrm{P}$, and $\mathrm{C}_{44}-\mathrm{P}$ are greater than zero, as shown in Figure 2A. This means that the mechanical properties of $\mathrm{CoCrScSn}$ compound in this lattice constant range are stable. One can see from Figure 2A that when the lattice constant is too large, it may cause negative values of $\mathrm{C}_{11}-\mathrm{C}_{12}-2 \mathrm{P}, \mathrm{C}_{11}+2 \mathrm{C}_{12}+\mathrm{P}$, and $\mathrm{C}_{44}-\mathrm{P}$. At this time, $\mathrm{CoCrScSn}$ compound will show unstable mechanical properties. This result is consistent with the fact that the interaction between atoms weakens and the stability is destroyed when the crystal expands. Under GGA $+\mathrm{U}$, the 

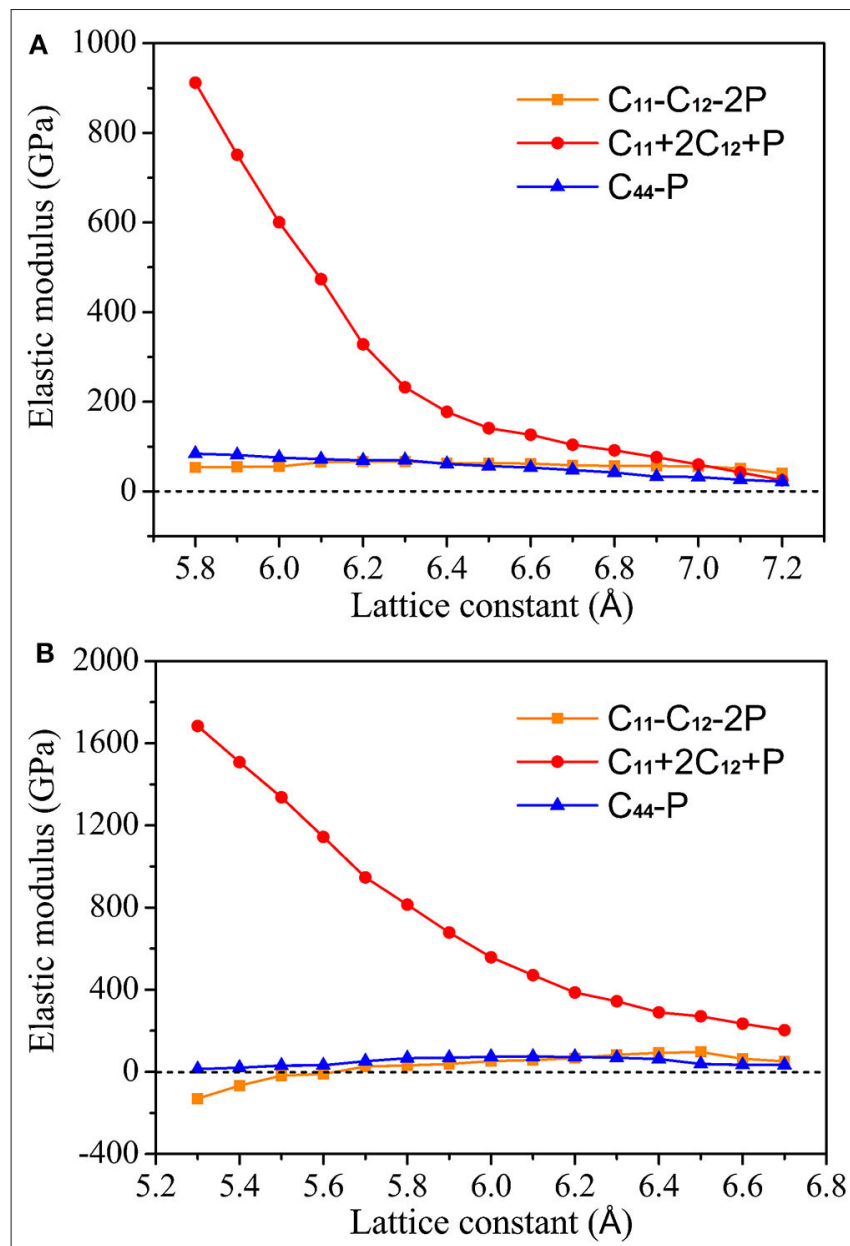

FIGURE 2 | Elastic modulus of CoCrScSn compound under different lattice constant. (A) GGA, (B) GGA + U.

mechanical properties of $\mathrm{CoCrScSn}$ are stable in the range of 5.66.7 $\AA$ as shown in Figure 2B. When the lattice constant is lower than $5.6 \AA, \mathrm{C}_{11}-\mathrm{C}_{12}-2 \mathrm{P}$ appears a negative value, and when the lattice constant is $>6.7 \AA, \mathrm{C}_{44}$-P may be a negative value, both of which will make the mechanical properties of the $\mathrm{CoCrScSn}$ compound unstable.

\section{Electronic Properties}

We further calculated the band structures of the CoCrScSn compound under GGA and GGA + U, as shown in Figures 3, 4, respectively. Under the cases of GGA and GGA $+U$, the majority-spin direction shows metal behavior, while minorityspin direction shows semiconductor behavior, which is a typical half-metal character. Furthermore, the band gap of minority-spin is an indirect under GGA, and direct (at the G point) under GGA + U. Under GGA and GGA + U, the minority-spin band gap of the title compound is 0.66 and $0.93 \mathrm{eV}$, respectively. The band gap considering Coulomb interaction is larger than that without Coulomb interaction. It shows that the Coulomb interaction has an obvious influence on the electronic structure of the CoCrScSn

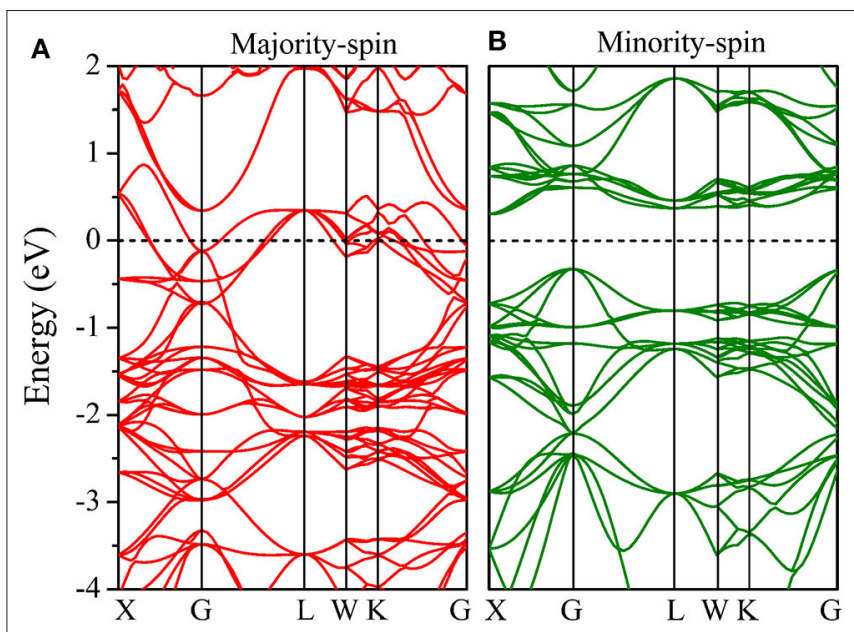

FIGURE 3 | Band structures of CoCrScSn compound under GGA. (A) Majority-spin, (B) minority-spin.

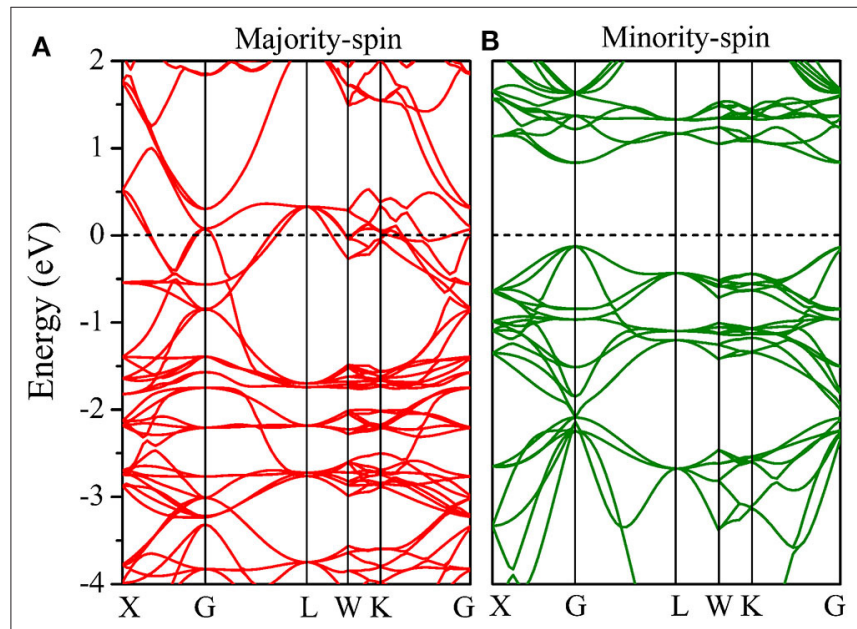

FIGURE 4 | Band structures of CoCrScSn compound under GGA + U. (A) Majority-spin, (B) minority-spin.

compound, so it is necessary to consider the Coulomb interaction when studying the electronic structure of the title compound.

Figure 5 shows the total and local density of states (DOSs) of CoCrScSn compound under GGA and GGA + U, respectively. Same as the result of the energy band structure, one spin direction is metallic, the other is semiconductor, and the title compound is a half-metal. By comparing the DOS distribution under GGA and GGA + U, it is clear that the shape of density of state in the two cases is roughly the same, but the local position is slightly different due to the Coulomb interaction. Obviously, the majority-spin below the Fermi level moves slightly to the low energy region, the minority-spin below the Fermi level moves toward the Fermi level, and the minority-spin above the Fermi level moves away from the Fermi level when the Coulomb interaction is considered. 


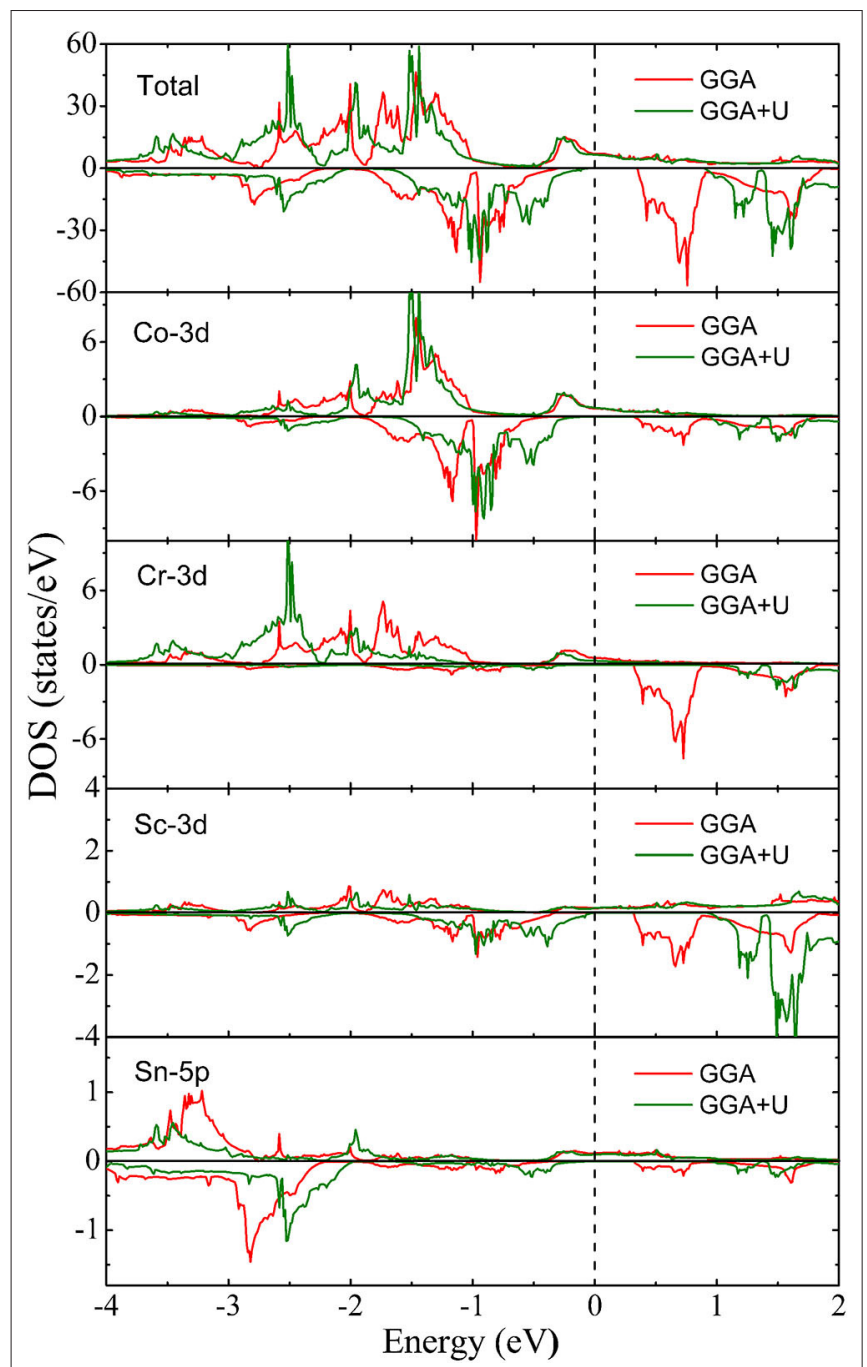

FIGURE 5 | Total and local density of states of CoCrScSn compound under $\mathrm{GGA}$ and $\mathrm{GGA}+\mathrm{U}$, respectively.

The robustness of half-metallicity is a key factor for spintronic materials. For practical applications, it is particularly important whether the half-metallic properties can still exist under external pressure or strain. Next, we will study the stability of halfmetallicity of $\mathrm{CoCrScSn}$ compound when the lattice constant changes. Figure 6 shows the curve of the minority-spin band gap width with the lattice constant of the $\mathrm{CoCrScSn}$ compound under GGA and GGA + U. The results show that the majorityspin direction always shows the metallic property when the lattice constant changes, therefore, the half-metallic property is determined by the minority-spin band gap. That is to say, when there is a band gap in minority-spin, the title compound has halfmetallic property, when there is no band gap, the half-metallicity will disappears.

Figure 6 shows that under GGA and GGA + U, the conduction band minimum (CBM) and valence band maximum $(\mathrm{VBM})$ in minority-spin change with the lattice constant. It can
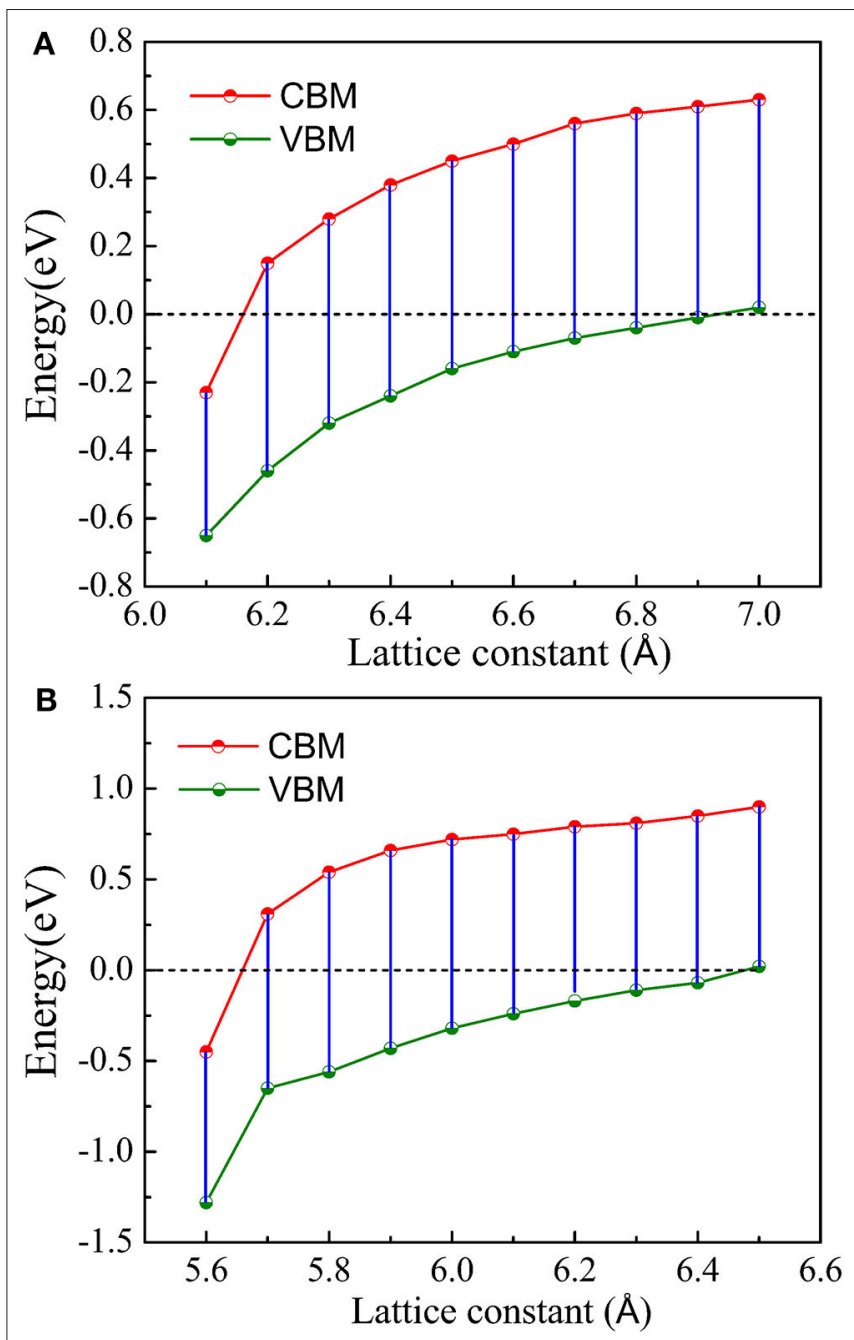

FIGURE 6 | Minority-spin CBM and VBM of CoCrScSn compound at different lattice constant. (A) GGA, (B) GGA + U.

be seen that within the lattice constants region of $6.2-6.9 \AA$, the CBM and VBM are located on the two sides of the Fermi level under GGA. At this time, the semiconductor character of minority-spin is obvious, the title compound has stable halfmetallic property. When the lattice constant is lower than $6.2 \AA$ or higher than $6.9 \AA$, the minority-spin band gap disappears and the half-metallicity disappears.

In the case of GGA $+\mathrm{U}$, the $\mathrm{CoCrScSn}$ compound also has similar characteristics, but due to the influence of Coulomb interaction, the lattice constant of the compound maintaining the half-metallicity region is from 5.7 to $6.4 \AA$. Combined with the mechanical properties research, we can find that in the case of GGA, the mechanical and half-metallic properties of the $\mathrm{CoCrScSn}$ compound are very stable within the lattice constant range of 6.2-6.9 $\AA$. In the case of GGA + U, the mechanical properties and half-metallic properties exist stably in the range of 5.7-6.4 . 


\section{Magnetic Properties}

Slater and Pauling in two pioneering papers show that if a minority-spin electron is added to a magnetic compound, the total magnetic moment of the compound will be reduced by $1 \mu_{\mathrm{B}}$. If a majority-spin electron is added, the total magnetic moment of the compound will be increased by $1 \mu_{\mathrm{B}}$ [55-57]. This behavior called Slater-Pauling (SP) rule has been studied not only in binary magnetic compounds [58], but also in halfmetallic Heusler compounds. So far, people have summarized some SP relation between the total magnetic moment $\left(M_{t}\right)$ and number of valence electrons $\left(Z_{t}\right)$ of per molecular unit in halfmetallic Heusler compounds, such as $M_{t}=Z_{t}-18$ [59], $M_{t}=$ $\mathrm{Z}_{\mathrm{t}}-24$ [4], and $\mathrm{M}_{\mathrm{t}}=\mathrm{Z}_{\mathrm{t}}-28$ [57]. Some half-metallic quaternary Heusler compounds mainly satisfy the SP rule of $\mathrm{M}_{\mathrm{t}}=\mathrm{Z}_{\mathrm{t}}-18$ [60-63] and $\mathrm{M}_{\mathrm{t}}=\mathrm{Z}_{\mathrm{t}}-24$ [64-66].

In $\mathrm{CoCrScSn}$ compound, the number of valence electrons of $\mathrm{Co}, \mathrm{Cr}, \mathrm{Sc}$ and $\mathrm{Sn}$ atoms are nine, six, three, and four, respectively, so the $Z_{t}$ is twenty two. The calculations of GGA and GGA + $U$ show that the $M_{t}$ at equilibrium lattice constant is $4.0 \mu_{B}$. Obviously, the $\mathrm{M}_{t}$ and $\mathrm{Z}_{\mathrm{t}}$ are in accordance with the SP relation $\mathrm{M}_{\mathrm{t}}=\mathrm{Z}_{\mathrm{t}}-18$, and CoCrScSn compound conform to the typical characteristics of half-metallic Heusler compound.

Figure 7 shows the total and local magnetic moments of $\mathrm{CoCrScSn}$ compound at different lattice constants under GGA and GGA + U, respectively. From 6.2 to $6.9 \AA$ under GGA and from 5.7 to $6.4 \AA$ under $\mathrm{GGA}+\mathrm{U}$, the $\mathrm{M}_{\mathrm{t}}$ is an integral value $4.0 \mu_{\mathrm{B}}$. Within this lattice constant range, the CoCrScSn compound has stable half-metallic character. When the lattice constant under GGA is lower than $6.2 \AA$ or higher than $6.9 \AA$, the $\mathrm{M}_{\mathrm{t}}$ is no longer an integral value, and the half-metallicity of the $\mathrm{CoCrScSn}$ compound will disappear.

The same situation also occurs when considering the Coulomb interaction. If the lattice constant is $<5.7 \AA$ or higher than $6.4 \AA$, the $M_{t}$ does not meet the SP rule and the title compound will lose the half-metal character. It can be seen from the local magnetic moment distribution curve that $\mathrm{Cr}$ atom is the most important source of the magnetic properties of the title compound, and Co atom also contributes to the total magnetic moment, but the contribution degree is smaller than $\mathrm{Cr}$ atom. It seems that $\mathrm{Sc}$ and $\mathrm{Sn}$ atoms also have certain magnetic moments, but because Sc and Sn atoms are not magnetic themselves, they are induced magnetic moments due to the influence of $\mathrm{Cr}$ and Co atoms.

Higher Curie temperature is another key factor for the application of half-metallic magnetic materials in spintronics. Theoretically, the mean field approximation is often used to estimate the Curie temperature $\left(T_{c}\right)$ of materials. The formula can be expressed as follows [37, 67]:

$$
T_{c}=\frac{2 \Delta E_{A F M-F M}}{3 C K_{B}}
$$

where $\triangle E_{A F M-F M}$ is the total energy difference between antiferromagnetic and ferromagnetic states. $C$ is the amount of the magnetic ion, and $k_{B}$ is Boltzmann constant. The Curie temperature calculated based on formula (6) for $\mathrm{CoCrScSn}$
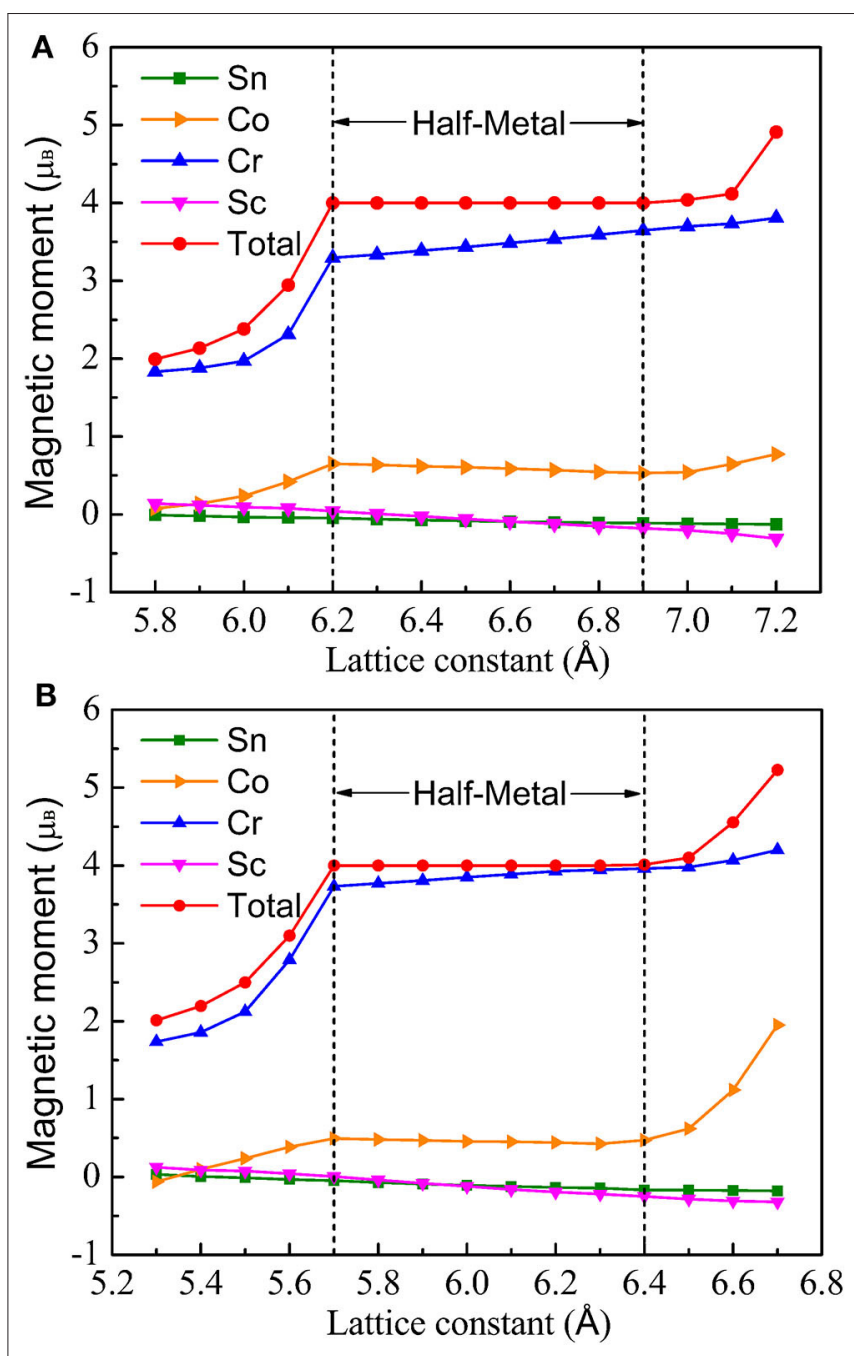

FIGURE 7 | Total and atomic magnetic moments of CoCrScSn compound at different lattice constant. (A) GGA, (B) GGA + U.

is $762 \mathrm{~K}$, which is slightly less than the previous theoretical research result.

Furthermore, Wurmehl et al. [68] proposed a linear relation to estimate the Curie temperature of the half-metal Heusler compound by using the $\mathrm{M}_{\mathrm{t}}$, and the expression is [69]

$$
T_{c}=23+181 M_{t}
$$

The Curie temperature for $\mathrm{CoCrScSn}$ were estimated according to the model presented is $747 \mathrm{~K}$, which is very close to the result of mean field approximation. Although the theoretical estimate of the Curie temperature is often higher than the actual value, we conclude that the Curie temperature of $\mathrm{CoCrScSn}$ should be higher than room temperature, which make it interesting for technological applications in spintronics. 


\section{CONCLUSIONS}

In this study, we focused on quaternary Heusler compound $\mathrm{CoCrScSn}$, and showed a complete first-principles study on the structure, mechanical, electronic, magnetic, and half-metallic properties of this material. It was found that the type-I structure of quaternary Heusler compound $\mathrm{CoCrScSn}$ is the most stable, and the type-I structure has a stable ferromagnetic ground state. Elastic constant analysis shows that $\mathrm{CoCrScSn}$ compound have stable mechanical properties at the equilibrium lattice constant. The mechanical properties of $\mathrm{CoCrScSn}$ will change if the lattice constant changes. The further studies show that if the lattice constant can be maintained within the range of 5.8-7.2 $\AA$ under GGA, and 5.6-6.7 $\AA$ under GGA + U, the mechanical properties of $\mathrm{CoCrScSn}$ compound are also stable. At the equilibrium lattice constant, the electronic structure of $\mathrm{CoCrScSn}$ compound under GGA and GGA + U shows that it has obvious half-metal character. The half-metal robustness of the CoCrScSn compound can be maintained in the lattice constant range of 6.2-6.9 $\AA$ for GGA, 5.7-6.4 $\AA$ for GGA + U. At the equilibrium lattice constant, the molecular magnetic moment of the compound is an integer value of $4.0 \mu_{\mathrm{B}}$. No matter how the lattice constant changes, as long as the compound has half-metallic character, the total magnetic moment of the CoCrScSn compound meets

\section{REFERENCES}

1. Wolf SA, Awschalom DD, Buhrman RA, Daughton JM, von Molnar S, Roukes ML, et al. Spintronics: a spin-based electronics vision for the future. Science. (2001) 294:1488-95. doi: 10.1126/science. 1065389

2. Zutic I, Fabian J, Das Sarma S. Spintronics: fundamentals and applications, Rev Mod Phys. (2004) 76:323-410. doi: 10.1103/RevModPhys.76.323

3. Pickett WE, Moodera JS. Half Metallic Magnets. Phys Today. (2001) 54:3944. doi: $10.1063 / 1.1381101$

4. Galanakis I, Dederichs PH, Papanikolaou N. Slater-Pauling behavior and origin of the half metallicity of the full-Heusler alloys. Phys Rev B. (2002) 66:174429. doi: 10.1103/PhysRevB.66.174429

5. Song Y, Dai G. Spin filter and spin valve in ferromagnetic graphene. Appl Phys Lett. (2015) 106:223104. doi: 10.1063/1.4921668

6. Bhat TM, Gupta DC. Robust thermoelectric performance and high spin polarisation in CoMnTiAl and FeMnTiAl compounds. RSC Adv. (2016) 6:80302-9. doi: 10.1039/c6ra18934b

7. Gupta DC, Bhat IH. Magnetic, electronic, high-spin polarization and half-metallic properties of $\mathrm{Ru}_{2} \mathrm{VGe}$ and $\mathrm{Ru}_{2} \mathrm{VSb}$ Heusler alloys: An FP-LAPW study. J Magn Magn Mater. (2015) 374:209-13. doi: 10.1016/j.jmmm.2014.08.032

8. Addadi Z, Doumi B, Mokaddem A, Elkeurti A, Sayede A, Tadjer A, et al. Electronic and ferromagnetic properties of $3 \mathrm{~d}(\mathrm{~V})$-doped $(\mathrm{BaS})$ barium sulfide. J Supercond Nov Magn. (2017) 30:917-23. doi: 10.1007/s10948-016-3894-3

9. Dahmane F, Tadjer A, Doumia B, Mesria D, Aouragb H, Sayede A. First principles study of the electronic structures and magnetic properties of transition metal-doped cubic indium nitride. Mater Sci Semicond Process. (2014) 21:66-73. doi: 10.1016/j.mssp.2014.01.037

10. Huang HM, Cao ML, Jiang ZY, Xiong YC, Zhang X, Luo SJ, et al. High spin polarization in formamidinium transition metal iodides: first principles prediction of novel half-metals and spin gapless semiconductors. Phys Chem Chem Phys. (2019) 21:16213. doi: 10.1039/c9cp00958b

11. De Groot RA, Mueller FM, Van Engen PG, Buschow KHJ. New class of materials: half-metallic ferromagnets. Phys Rev Lett. (1983) 50:2024. doi: 10.1103/PhysRevLett.50.2024 the Slater-Pauling rule. The mean field approximation shows that the Curie temperature is higher than room temperature, which makes the title compound a promising application in spintronics.

\section{DATA AVAILABILITY STATEMENT}

All datasets generated for this study are included in the article/supplementary material.

\section{AUTHOR CONTRIBUTIONS}

$\mathrm{CZ}$ designed the research. $\mathrm{HH}$ analyzed the data and results. $\mathrm{CZ}$ and $\mathrm{HH}$ performed the research and wrote the paper. $\mathrm{CW}$ checked the article grammar. ZH, ZZ, and GL contributed to the revisions. All authors contributed to the article and approved the submitted version.

\section{FUNDING}

This work was supported by the Natural Science Foundation of Hubei Province (No. 2017CFB740) and the Doctoral Scientific Research Foundation of Hubei University of Automotive Technology (No. BK201804).
12. Huang HM, Luo SJ, Yao KL. Half-metallicity and tetragonal distortion in semiheusler alloy FeCrSe. J Appl Phys. (2014) 115:043713. doi: 10.1063/1.4863122

13. Han Y, Bouhemadou A, Khenata R, Cheng Z, Yang T, Wang X. Prediction of possible martensitic transformations in all-d-metal zinc-based heusler alloys from first-principles. J Magn Magn Mater. (2019) 471:4955. doi: 10.1016/j.jmmm.2018.09.053

14. Chen Y, Chen S, Wang B, Wu B, Huang H, Qin X, et al. Half-Metallicity and magnetism of the quaternary Heusler compound $\mathrm{TiZrCoIn}_{1-\mathrm{x}} \mathrm{Ge}_{\mathrm{x}}$ from the first-principles calculations. Appl Sci. (2019) 9:620. doi: 10.3390/app9040620

15. Tan X, You J, Liu PF, Wang Y. Theoretical study of the electronic, magnetic, mechanical and thermodynamic properties of the spin gapless semiconductor CoFeMnSi. Crystals. (2019) 9:678. doi: 10.3390/cryst9120678

16. Li T, Wu Y, Han Y, Wang X. Tuning the topological nontrivial nature in a family of alkali-metal-based inverse heusler compounds: a first-principles study. J Magn Magn Mater. (2018) 463:44-9. doi: 10.1016/j.jmmm.2018.05.025

17. Feng L, Ma J, Yang Y, Lin T, Wang L. The electronic, magnetic, halfmetallic and mechanical properties of the equiatomic quaternary heusler compounds FeRhCrSi and FePdCrSi: a first-principles study. Appl Sci. (2018) 8:2370. doi: 10.3390/app8122370

18. Sakon T, Yamasaki Y, Kodama H, Kanomata T, Nojiri H, Adachi Y. The characteristic properties of magnetostriction and magneto-volume effects of $\mathrm{Ni}_{2} \mathrm{MnGa}$-Type ferromagnetic heusler alloys. Materials. (2019) 12:3655. doi: $10.3390 / \mathrm{ma1} 2223655$

19. Faleev SV, Ferrante Y, Jeong J, Samant MG, Jones B, Stuart P. Origin of the tetragonal ground state of Heusler compounds. Phys Rev Appl. (2017) 7:034022. doi: 10.1103/PhysRevApplied.7.034022

20. Wang XT, Dai XF, Wang LY, Liu XF, Wang WH, Wu GH, et al, Electronic structures and magnetism of $\mathrm{Rh}_{3} \mathrm{Z}(\mathrm{Z}=\mathrm{Al}, \mathrm{Ga}$, In, $\mathrm{Si}, \mathrm{Ge}$, $\mathrm{Sn}, \mathrm{Pb}, \mathrm{Sb}$ ) with $\mathrm{DO}_{3}$ structures. J Magn Magn Mater. (2015) 378:1623. doi: $10.1016 /$ j.jmmm.2014.10.161

21. Dahmane F, Mesri D, Tadjer A, Khenata R, Benalia S, Djoudi L, et al. Electronic structure, magnetism and stability of $\mathrm{Co}_{2} \mathrm{CrX}(\mathrm{X}=\mathrm{Al}, \mathrm{Ga}, \mathrm{In})$ ab initio study. Mod Phys Lett B. (2016) 30:1550265. doi: 10.1142/S0217984915502656

22. Han YL, Wu MX, Kuang MQ, Yang T, Chen XB, Wang XT. All-d-metal equiatomic quaternary Heusler hypothetical alloys ZnCdTMn $(\mathrm{T}=\mathrm{Fe}, \mathrm{Ru}$, $\mathrm{Os}, \mathrm{Rh}, \mathrm{Ir}, \mathrm{Ni}, \mathrm{Pd}, \mathrm{Pt}$ ): a first-principle investigation of electronic structures, 
magnetism, and possible martensitic transformations. Results Phys. (2018) 11:1134-41. doi: 10.1016/j.rinp.2018.11.024

23. Li TZ, Khenata $\mathrm{R}$, Cheng ZX, Chen $\mathrm{H}$, Yuan HK, Yang $\mathrm{T}$, et al. Martensitic transformation, electronic structure and magnetism in D03ordered heusler $\mathrm{Mn}_{3} \mathrm{Z}(\mathrm{Z}=\mathrm{B}, \mathrm{Al}, \mathrm{Ga}, \mathrm{Ge}, \mathrm{Sb})$ alloys, Acta Cryst. (2018) B74:673-80. doi: 10.1107/S2052520618013525

24. Berri S. First-principles calculations to investigate the structural, electronic, and half-metallic properties of $\mathrm{Ti}_{2} \mathrm{RhSn}_{1-\mathrm{x}} \mathrm{Si}_{\mathrm{x}}, \mathrm{Ti}_{2} \mathrm{RhSn}_{1-\mathrm{x}} \mathrm{Ge}_{\mathrm{x}}$, and $\mathrm{Ti}_{2} \mathrm{RhGe}_{1-\mathrm{x}} \mathrm{Si}_{\mathrm{x}}(\mathrm{x}=0,0.25,0.5,0.75$, and 1$)$ quaternary heusler alloys. $J$ Supercond Nov Magn. (2019) 32:2219-28. doi: 10.1007/s10948-018-4952-9

25. Khalaf Al-zyadi JM, Kadhim AA, Yao KL. Half-metallicity of the (001), (111) and (110) surfaces of CoRuMnSi and interface half-metallicity of CoRuMnSi/CdS. RSC Adv. (2018) 8:25653. doi: 10.1039/c8ra02918k

26. Huang H, Yang K, Zhao W, Zhou T, Yang X, Wu B. High-pressure-induced transition from ferromagnetic semiconductor to spin gapless semiconductor in quaternary heusler alloy $\mathrm{VFeScZ}$ ( $\mathrm{Z}=\mathrm{Sb}$, As, P). Appl Sci. (2019) 9:2859. doi: 10.3390/app9142859

27. Jin Y, Kharel P, Lukashev P, Valloppilly S, Staten B, Herran J, et al. Magnetism and electronic structure of $\mathrm{CoFeCrX}(\mathrm{X}=\mathrm{Si}, \mathrm{Ge})$ heusler alloys. J Appl Phys. (2016) 120:053903. doi: 10.1063/1.4960350

28. Chen $\mathrm{Z}, \mathrm{Xu} \mathrm{H}$, Gao Y, Wang $\mathrm{X}$, Yang T. Site-Preference, electronic, magnetic, and half-metal properties of full-heusler $\mathrm{Sc}_{2} \mathrm{VGe}$ and a discussion on the uniform strain and tetragonal deformation effects. Crystals. (2019) 9:445. doi: 10.3390/cryst9090445

29. Wang XT, Zhao WQ, Cheng ZX, Dai XF, Khenata R. Electronic, magnetic, halfmetallic and mechanical properties of a new quaternary heusler compound ZrRhTiT1: Insights from first-principles studies. Solid State Commun. (2018) 269:125-30. doi: 10.1016/j.ssc.2017.10.021

30. Zhang HP, Liu WB, Lin TT, Wang WH, Liu GD. Phase stability and magnetic properties of $\mathrm{Mn}_{3} \mathrm{Z}(\mathrm{Z}=\mathrm{Al}, \mathrm{Ga}, \mathrm{In}, \mathrm{Tl}, \mathrm{Ge}, \mathrm{Sn}, \mathrm{Pb})$ heusler alloys. Appl Sci. (2019) 9:964. doi: 10.3390/app9050964

31. Pourebrahim G, Ahmadian F, Momeni P. Robust half-metallicity in quaternary heusler compounds $\mathrm{KSrNZ}(\mathrm{Z}=\mathrm{O}$, S, and Se). J Supercond Novel Magn. (2019) 32:3305-14. doi: 10.1007/s10948-019-5098-0

32. Yousuf S, Gupta DC. Insight into half-metallicity, spin-polarization and mechanical properties of $\mathrm{L}_{1}$ structured $\mathrm{MnY}_{2} \mathrm{Z} \quad(\mathrm{Z}=\mathrm{Al}$, $\mathrm{Si}, \mathrm{Ga}, \mathrm{Ge}, \mathrm{Sn}$, Sb) heusler alloys. J Alloy Compd. (2018) 735:1245-52. doi: 10.1016/j.jallcom.2017.11.239

33. Benea D, Gavrea R, Coldea M, Isnard O, Pop V. Half-metallic compensated ferrimagnetism in the Mn-Co-V-Al Heusler alloys. J Magn Magn Mater. (2019) 475:229-33. doi: 10.1016/j.jmmm.2018.11.115

34. Li Y, Liu GD, Wang XT, Liu EK, Xi XK, Wang WH, et al. Firstprinciples study on electronic structure, magnetism and half-metallicity of the NbCoCrAl and NbRhCrAl compounds. Results Phys. (2017) 7:224854. doi: 10.1016/j.rinp.2017.06.047

35. Gökoglu G. Ab initio electronic structure of NiCoCrGa halfmetallic quaternary heusler compound. Solid State Sci. (2012) 14:1273-6. doi: 10.1016/j.solidstatesciences.2012.07.013

36. Xu G, You Y, Gong Y, Liu E, Xu F, Wang W. Highly-dispersive spin gapless semiconductors in rare-earth-element contained quaternary heusler compounds. J Phys D Appl Phys. (2017) 50:105003. doi: 10.1088/1361-6463/aa57a3

37. Wu B, Huang H, Zhou G, Yang X, Li P, Feng Y. Half-metallic ferrimagnets behavior of a new quaternary Heusler alloy $\operatorname{CrCoScZ}(Z=S i, G e, S n)$. Eur Phys J B. (2019) 92:119. doi: 10.1140/epjb/e2019-90646-4

38. Huang HM, Jiang ZY, Hu YJ, Li W, Luo S. Effect of coulomb interaction on the electronic properties of bulk and surface of quaternary heusler $\mathrm{CoCrScBi}$. Solid State Sci. (2019) 97:106018. doi: 10.1016/j.solidstatesciences.2019. 106018

39. Khan MI, Arshad H, Rizwan M, Gillani SSA, Zafar M, Ahmed S, et al. Investigation of structural, electronic, magnetic and mechanical properties of a new series of equiatomic quaternary Heusler alloys CoYCrZ ( $\mathrm{Z}=\mathrm{Si}, \mathrm{Ge}, \mathrm{Ga}, \mathrm{Al})$ : a DFT study. J Alloy Comp. (2020) 819:152964. doi: 10.1016/j.jallcom.2019.152964

40. Gao GY, Hu L, Yao KL, Luo B, Liu N. Large half-metallic gaps in the quaternary heusler alloys $\mathrm{CoFeCrZ} \mathrm{(Z=Al,} \mathrm{Si,} \mathrm{Ga,} \mathrm{Ge):} \mathrm{a} \mathrm{first-principles} \mathrm{study.} \mathrm{J} \mathrm{Alloy}$ Comp. (2013) 551:539-43. doi: 10.1016/j.jallcom.2012.11.077
41. Paudel R, Zhu J. Structural, electronic, magnetic, elastic, and thermal properties of co-based equiatomic quaternary Heusler alloys. J Magn Magn Mater. (2018) 453:10-6. doi: 10.1016/j.jmmm.2017.12.103

42. Perdew JP, Burke K, Ernzerhof M. Generalized gradient approximation made simple. Phys Rev Lett. (1996) 77:3865-8. doi: 10.1103/PhysRevLett.77.3865

43. Kresse G, Furthmüller J. Efficiency of ab-initio total energy calculations for metals and semiconductors using a plane-wave basis set. Comput Mater Sci. (1996) 6:15-50. doi: 10.1016/0927-0256(96)00008-0

44. Rai DP, Thapa RK. An investigation of semiconducting behavior in the minority spin of $\mathrm{Co}_{2} \mathrm{CrZ}(\mathrm{Z}=\mathrm{Ga}, \mathrm{Ge}, \mathrm{As})$ : LSDA and LSDA+U method. $J$ Alloy Comp. (2012) 542:257-63. doi: 10.1016/j.jallcom.2012.07.059

45. Rai DP, Thapa RK. An abinitio study of the half-metallic properties of $\mathrm{Co}_{2} \mathrm{TGe}(\mathrm{T}=\mathrm{Sc}, \mathrm{Ti}, \mathrm{V}, \mathrm{Cr}, \mathrm{Mn}, \mathrm{Fe})$ : LSDA+U method. J Korean Phys Soc. (2013) 62:1652-60. doi: 10.3938/jkps.62.1652

46. Curtarolo S, Setyawan W, Hart GLW, Jahnatek M, Chepulskii RV, Taylor RH, et al. AFLOW: An automatic framework for high-throughput materials discovery. Comput Mater Sci. (2012) 58:218-26. doi: 10.1016/j.commatsci.2012.02.005

47. Idrissia S, Khalladia R, Zitib S, El Mekkaouia N, Mtouguia S, Labrimc H, et al. The electronic and magnetic proprieties of the rare earth-based quaternary heusler compound LuCoVGe. Physica B Condens Matter. (2019) 562:11623. doi: 10.1016/j.physb.2019.03.018

48. Rani D, Bainsla L, Suresha KG, Alam A. Experimental and theoretical investigation on the possible half-metallic behaviour of equiatomic quaternary heusler alloys: CoRuMnGe and CoRuVZ $(\mathrm{Z}=\mathrm{Al}, \mathrm{Ga})$. J Magn Magn Mater. (2019) 492:165662. doi: 10.1016/j.jmmm.2019.165662

49. Forozani G, Abadi AAM, Baizaee SM, Gharaati A. Structural, electronic and magnetic properties of CoZrIrSi quaternary heusler alloy: first-principles study. J Alloy Comp. (2020) 815:152449. doi: 10.1016/j.jallcom.2019.152449

50. Wei XP, Gao PF, Zhang YL. Investigations on gilbert damping, curie temperatures and thermoelectric properties in $\mathrm{CoFeCrZ}$ quaternary heusler alloys. Curr Appl Phys. (2020) 20:593-603. doi: 10.1016/j.cap.2020.02.007

51. Mehmood N, Ahmad R, Murtaza G. Ab initio investigations of structural, elastic, mechanical, electronic, magnetic, and optical properties of half-heusler compounds RhCrZ (Z=Si, Ge). J Supercond Novel Magn. (2017) 30:24818. doi: 10.1007/s10948-017-4051-3

52. Huang YC, Guo XF, Ma YL, Shao HB, Xiao ZB. Stabilities, electronic and elastic properties of $\mathrm{Ll}_{2}-\mathrm{Al}_{3}\left(\mathrm{Sc}_{1-\mathrm{x}}, \mathrm{Zr}_{\mathrm{x}}\right)$ with different $\mathrm{Zr}$ content: a first-principles study. Physica B Condens Matter. (2018) 548:27-33. doi: 10.1016/j.physb.2018.08.019

53. Allali D, Bouhemadou A, Zerarga F, Ghebouli MA, Bin-Omran S. Prediction study of the elastic and thermodynamic properties of the $\mathrm{SnMg}_{2} \mathrm{O}_{4}$, $\mathrm{SnZn}_{2} \mathrm{O}_{4}$ and $\mathrm{SnCd}_{2} \mathrm{O}_{4}$ spinel oxides. Comput Mater Sci. (2012) 60:21723. doi: 10.1016/j.commatsci.2012.03.044

54. Gu JB, Wang CJ, Zhang WX, Sun B, Liu GQ, Liu DD, et al. High-pressure structure and elastic properties of tantalum single crystal: first principles investigation. Chin Phys B. (2016) 25:126103. doi: 10.1088/1674-1056/25/12/126103

55. Slater JC. The ferromagnetism of nickel. II. Temperature effects. Phys Rev. (1936) 49:931. doi: 10.1103/PhysRev.49.931

56. Pauling L. The nature of the interatomic forces in metals. Phys Rev. (1938) 54:899. doi: 10.1103/PhysRev.54.899

57. Skaftouros S, Özdogan K, Saşioglu E, Galanakis I. Generalized slaterpauling rule for the inverse heusler compounds. Phys Rev B. (2013) 87:024420. doi: 10.1103/PhysRevB.87.024420

58. Khalaf Al-zyadi JM, Samuel RM, Gao GY, Yao KL. The half-metallic characteristics of the (001) surface of zinc-blende TiTe. J Magn Magn Mater. (2013) 346:166-70. doi: 10.1016/j.jmmm.2013.07.032

59. Galanakis I, Dederichs PH, Papanikolaou N. Origin and properties of the gap in the half-ferromagnetic heusler alloys, Phys Rev B. (2002) 66:134428. doi: 10.1103/PhysRevB.66.134428

60. Berri S. Electronic structure and half-metallicity of the new heusler alloys PtZrTiAl, PdZrTiAl and Pt 0.5 Pd 0.5 ZrTiAl. Chin J Phys. (2017) 55:195202. doi: 10.1016/j.cjph.2016.10.017

61. Berri S, Ibrir M, Maouche D, Attallah M. Robust half-metallic ferromagnet of quaternary heusler compounds $\mathrm{ZrCoTiZ}(\mathrm{Z}=\mathrm{Si}, \mathrm{Ge}, \mathrm{Ga}$ and $\mathrm{Al})$. Comput Condens Matter. (2017) 1:26-31. doi: 10.1016/j.cocom.2014.10.003 
62. Berri S, Ibrir M, Maouche D, Attallah M. First principles study of structural, electronic and magnetic propertiesof $\mathrm{ZrFeTiAl}$, ZrFeTiSi, ZrFeTiGe and ZrNiTiAl. J Magn Magn Mater. (2014) 371:106-11. doi: 10.1016/j.jmmm.2014.07.033

63. Xie HH, Gao Q, Li L,Lei G, Mao GY, Hu XR, Deng JB. First-principles study of four quaternary heusler alloys $\mathrm{ZrMnVZ}$ and $\mathrm{ZrCoFeZ}(\mathrm{Z}=\mathrm{Si}, \mathrm{Ge})$. Comput Mater Sci. (2015) 103:52-5. doi: 10.1016/j.commatsci.2015.03.010

64. Berri S, Maouche D, Ibrir M, Zerarga F. A first-principle study of half-metallic ferrimagnetism in the CoFeTiSb quaternary heusler compound. J Magn Magn Mater. (2014) 354:65-9. doi: 10.1016/j.jmmm.2013.10.044

65. Berri S. First-principles study on half-metallic properties of the CoMnCrSb quaternary Heusler compound. J Supercond Nov Magn. (2016) 29:130915. 10.1007/s10948-016-3404-7

66. Khalaf Al-zyadi JM,Kadhim AA, Yao KL. Electronic and magnetic properties of the (001) surface of the CoNbMnSi heusler alloy: firstprinciples calculations. J Electron Spectrosc Relat Phenom. (2018) 226:1721. doi: 10.1016/j.elspec.2018.04.005

67. Rostamia M, Afkania M, Torkamania MR, Kanjouri F. Bulk and surface DFT investigations of the electronic and magnetic properties of CsXNO (X=Mg, Ca and Sr) quaternary heusler alloys. Mater Chem Phys. (2020) 248:122923. doi: 10.1016/j.matchemphys.2020.122923
68. Wurmehl S, Fecher GH, Kandpal HC, Ksenofontov V, Felser C, Lin HJ, et al., Geometric, electronic, and magnetic structure of $\mathrm{Co}_{2} \mathrm{FeSi}$ : Curie temperature and magnetic moment measurements and calculations. Phys Rev B. (2005) 72:184434. doi: 10.1103/PhysRevB.72. 184434

69. Candan A, Ugur G, Charifi Z, Baaziz H, Ellialtioglu MR. Electronic structure and vibrational properties in cobalt-based full-Heusler compounds: a first principle study of $\mathrm{Co}_{2} \mathrm{MnX}$ (X=Si, Ge, Al, Ga). J Alloy Comp. (2013) 560:21522. doi: 10.1016/j.jallcom.2013.01.102

Conflict of Interest: The authors declare that the research was conducted in the absence of any commercial or financial relationships that could be construed as a potential conflict of interest.

Copyright (c) 2020 Zhang, Huang, Wu, Zhu, He and Liu. This is an open-access article distributed under the terms of the Creative Commons Attribution License (CC $B Y)$. The use, distribution or reproduction in other forums is permitted, provided the original author(s) and the copyright owner(s) are credited and that the original publication in this journal is cited, in accordance with accepted academic practice. No use, distribution or reproduction is permitted which does not comply with these terms. 